\title{
Value of pathology review in a population-based series of ovarian tumors
}

Colin JR Stewart ${ }^{1,2}$, Louise M Stewart ${ }^{3}$, C D’Arcy J Holman ${ }^{4}$ Susan Jordan ${ }^{5}$, James Semmens ${ }^{3}$ Katrina Spilsbury ${ }^{3}$, Timothy Threlfall ${ }^{6}$

${ }^{1}$ Department of Pathology King Edward Memorial Hospital, Perth, ${ }^{2}$ School of Women's and Infants' Health, University of Western Australia, ${ }^{3}$ Center for Population Health Research, Curtin University, Western Australia, ${ }^{4}$ School of Population Health, University of Western Australia, ${ }^{5}$ Population Health Department, QIMR Berghofer Medical Research Institute, Brisbane, Queensland, and ${ }^{6}$ Western Australian Cancer Registry, Department of Health, Western Australia.

Running title: Review of ovarian tumors

\section{Conflicts of Interest and Sources of Funding:}

There are no conflicts of interest. This research was funded by the Ovarian Cancer Research Foundation (OCRF), Australia, and SJ is funded by a fellowship from the National Health and Medical Research Council of Australia.

Correspondence: Dr C Stewart, Department of Histopathology, King Edward Memorial Hospital, Bagot Road, Perth, Western Australia 6008. Telephone: 089340 2715, Fax: 089340 2636, Email: colin.stewart@health.wa.gov.au 


\section{ABSTRACT}

Ovarian neoplasia comprises a heterogenous group of tumors with distinct clinicopathologic and molecular features and therefore assessment of potential risk factors should be tumor subtypespecific. As part of on-going epidemiological investigations of ovarian neoplasia in Western Australia (WA), we performed an initial review of original pathology reports followed, in selected cases, by reassessment of histology material to optimize accurate diagnosis. Additional immunohistochemistry, often using antibodies unavailable at the time of initial assessment, was also performed as required. From an initial cohort of 1660 cases identified through the WA Cancer Registry (WACR), benign, non-epithelial, non-ovarian, miscellaneous and indeterminate cases were excluded. Also excluded were 33 cases that were reclassified as ovarian metastases rather than primary ovarian tumors. Following exclusions there remained 1321 borderline and malignant epithelial neoplasms. The diagnosis was considered accurate in 1186 cases $(89.8 \%)$ based upon information in the initial pathology reports and clinical follow-up data but uncertain in 135 cases $(10.2 \%)$. Histologic review was possible in 92 of the latter tumors leading to an amended diagnosis in 63 cases $(68.5 \%)$. The most common types of diagnostic amendment were the reclassification of high-grade carcinomas of undifferentiated, endometrioid or transitional appearance as high-grade serous carcinoma, and the reclassification of most carcinomas of mixed epithelial type as 'pure' carcinomas. This review illustrated specific pitfalls in the diagnosis of ovarian epithelial neoplasia and helped to maintain the accuracy of the WACR. Accurate diagnosis will optimise further epidemiological studies assessing risk factors in specific subtypes of ovarian neoplasia. 


\section{INTRODUCTION}

Epidemiological studies play a key role in public health analysis including the identification of factors that predispose to, or may be protective towards, the development of certain tumors. In the gynecological context, for example, the risk of ovarian, fallopian tube and primary peritoneal (hereafter ovarian) neoplasia has been positively linked to certain hereditary factors (BRCA mutations and Lynch syndrome), infertility, pelvic inflammatory disease and in vitro fertilization, whereas hormonal contraception, parity and prior salpingectomy or tubal ligation appear to have a protective role (1-6).

A limitation of some epidemiological studies of ovarian neoplasia, particularly those conducted more than a decade ago, is that in many cases malignant epithelial tumors were grouped together and regarded as a single entity (epithelial ovarian cancer), and occasionally the same has applied to the analysis of borderline tumours. However, it is now recognized that ovarian tumors comprise a heterogenous group of neoplasms with differing clinical, histologic, immunophenotypic and molecular characteristics, and hence are likely to have different etiology (7-10). There are two major categories of borderline ovarian tumor (serous and mucinous) with a much less common subgroup exhibiting mixed epithelial (seromucinous) differentiation, and there are five major subtypes of ovarian carcinoma namely high-grade serous carcinoma (HGSC), low-grade serous carcinoma (LGSC), endometrioid carcinoma (EC), clear cell carcinoma (CCC), and mucinous carcinoma (MUCA). Carcinosarcoma, while less common, is also now regarded as a form of metaplastic carcinoma most often showing high-grade serous differentiation in its epithelial component (9). High-grade serous carcinoma accounts for approximately $65-70 \%$ of all ovarian carcinomas and hence studies of epithelial ovarian cancer not otherwise specified (NOS) are heavily biased towards this tumor subtype. This has major 
implications regarding the investigation of etiological factors, possible preventative measures, and the optimal management of ovarian carcinoma since the identification of risk factors and treatment responses in the less common tumor subtypes may be obscured by the predominance of HGSC (11).

An additional difficulty presented by epidemiological analysis of relatively uncommon tumors such as ovarian carcinoma is that such studies generally cover a broad time period. This has the benefit of increasing the size of patient cohorts, thus maximising the power of statistical analysis. However, older histopathology diagnoses may not adhere to currently recognized tumor classifications and may lack the benefit of more recently developed ancillary diagnostic techniques such as immunohistochemistry (IHC) or molecular analysis $(12,13)$. Furthermore, there may be significant changes in the interpretation of specific diagnostic scenarios, for example the appreciation that many tumors previously classified as high-stage ovarian MUCA are likely to represent primary non-gynecological malignancies metastatic to the ovary $(14,15)$.

In a previous population-based study performed in Western Australia (WA) we found that women who had undergone hysterectomy with or without unilateral salpingo-oophorectomy (USO) had a reduced risk of ovarian cancer, confirming earlier reports (3). Unexpectedly, however, patients who had undergone USO without hysterectomy had a four-fold increased risk of ovarian cancer. This study has since been extended to include a larger cohort of approximately 850,000 women in WA followed up for up to 45 years. Their detailed demographic, reproductive and surgical data obtained via linkage of routinely collected hospital, births and cancer registry information will be used to further clarify risk factors associated with the development of borderline or malignant epithelial ovarian neoplasms. However, in view of the potential limitations in tumor classification discussed above it was considered beneficial that pathology 
review should be performed for identified cases, comprising at a minimum evaluation of the original diagnostic reports with re-examination of the original histologic material if required. In this report we discuss the results of this pathologic review, discussing amendments to the original diagnoses and the effects such review might have on epidemiological analysis.

\section{MATERIALS AND METHODS}

The study group comprised all women born in WA between 1945 and 1975 recorded in the WA Cancer Registry (WACR) to have a new diagnosis of borderline or malignant ovarian tumor between January 1982 and the end of June 2014. Given the heterogeneity of ovarian neoplasia one important aspect of this study was to ensure, as far as possible, that the tumors were correctly assigned in terms of pathological diagnosis. Therefore the pathology reports of the ovarian tumors (and those of any relevant earlier or subsequent cytology, biopsy or resection specimens) were reviewed by a gynecological pathologist (CJRS). In some cases, clinical followup data were used to aid diagnostic categorization. For example, in cases of MUCA, long-term patient survival and the absence of any subsequently identified extra-ovarian mucinous neoplasm were considered strong support for the primary nature of the ovarian tumor. Cases in which the pathology report was unavailable were excluded. For the remaining cases, based upon the clinical information, the microscopic description of the tumor, the results of any immunohistochemical and molecular analysis, and available follow-up data, the original diagnosis was determined to be either confirmed or uncertain. The latter category included specific diagnostic scenarios (outlined below) which are known to cause difficulty in the diagnosis or subtyping of ovarian neoplasia. Review of the original histologic and/or cytologic slides was performed in these cases. If review of tumors with uncertain diagnosis was impossible 
then the cases were excluded. Additional IHC was performed in selected cases, often using antibodies that were not available at the time of initial diagnosis. In the histologically reviewed (uncertain) cases it was determined whether the original diagnosis was confirmed or amended, and the contribution of any additional IHC was also evaluated.

The indications for histologic review included the following: tumors initially reported as 'papillary adenocarcinoma' or carcinoma/ adenocarcinoma, NOS; bilateral tumors other than those of serous type; tumors in which there was earlier or subsequent identification of an extraovarian neoplasm that may have been confused with a primary ovarian tumor (particularly mucinous and endometrioid carcinoma); all cases reported as grade $3 \mathrm{EC}$, primary transitional cell carcinoma (TCC), or carcinoma of mixed histologic type; cases in which there appeared to be a possible discrepancy between the microscopic description of the tumor, the results (or absence) of appropriate ancillary studies, and/or clinical follow-up information.

During the study period some serous carcinomas had been reported initially as moderately differentiated/ grade 2, based upon the 'universal' grading system for ovarian neoplasia (16) It is now accepted that HGSC and LGSC are different entities rather than different grades of one tumor type and thus the intermediate grade category for serous carcinoma is no longer applicable (17). In the current review it was clear from the original pathology reports that most serous carcinomas initially reported to be grade 2 were HGSC and these were reclassified as such. However, cases in which the distinction of HGSC and LGSC was impossible were reviewed.

The current pathologic review concentrated on the major subtypes of borderline and malignant epithelial tumors since these are the main focus of on-going epidemiological investigations. Therefore sex cord-stromal neoplasms, malignant germ cell tumors and other rare 
miscellaneous tumors were not examined in detail. However, cases with possible discordant diagnoses were reviewed whenever possible following the guidelines above. Furthermore, many of the tumors in these categories (particularly sex cord- stromal tumors) have been reviewed during recent unrelated studies (18-20).

The study received ethics approval (WA Department of Health HREC \#2015/07, Curtin University HREC HR 41/2015).

\section{RESULTS}

In total, 1660 women were identified from the WACR database during the study period. The original pathology reports were unavailable in 42 cases and two spurious cases were identified (one a duplicated entry and the other a non-WA case that was reviewed as part of an independent study); these were excluded from further analysis. There were 84 malignant sex cord-stromal tumors, 56 malignant germ cell tumors and 25 miscellaneous rare neoplasms. Of the remaining 1451 cases, 24 were considered to be benign and 33 were favoured to represent metastases to the ovary following reassessment. Twenty-one tumors remained of indeterminate subtype or origin usually because of limited diagnostic material. Fifty-two cases were excluded since they did not represent primary ovarian neoplasms. These included pathologically unrelated tumors such as retroperitoneal sarcomas which were captured in the initial search due to overlapping codes with ovarian neoplasia in the WACR. The process of case review and exclusion is outlined in Figure 1.

Histologic and/or cytologic review was performed in 28 of the excluded tumors leading to a revision of the original diagnosis in 23 cases (82.1\%), most commonly resulting in a final diagnosis of metastatic carcinoma rather than a primary ovarian carcinoma ( $\mathrm{n}=14$, Table 1$)$. In 
the latter cases the tumor origin was favored to be from the gastrointestinal tract, cervix and endometrium in nine, three and two cases, respectively, and the amended diagnoses were supported by additional immunohistochemical studies in 10 cases (Figs. 2 and 3).

Following the selection process described above 1321 borderline and malignant epithelial tumors of major subtypes remained in the main study group (Figure 1). The initial diagnosis was considered to be accurate in $1186(89.8 \%)$ cases based upon the information presented in the original pathology report together with any relevant follow-up data. However, the diagnosis was considered uncertain in the remaining $135(10.2 \%)$ cases almost all of which $(n=129)$ were malignant. Slide review was performed in $92(68.1 \%)$ of the uncertain tumors but was impossible in the remaining $43(31.9 \%)$ cases because the original diagnostic slides were unavailable for examination. Following exclusion of the latter cases, there remained 1278 tumors including 419 borderline tumors and 859 carcinomas. The number of cases with histologic review, the number with amended diagnosis, and the use of additional IHC for each tumor subtype are summarized in Table 2.

Of the 92 uncertain cases that underwent histologic review, the original diagnosis was confirmed in $29(31.5 \%)$ cases but was amended in the remaining $63(68.5 \%)$ cases. The types of diagnostic amendment are summarized in Table 3. Across the histological spectrum, the most common causes of diagnostic amendment were changes in grade (arguably tumor subtype rather than grade in serous carcinomas), tumor categorisation (for example grade 3 EC being revised to HGSC), and reclassification of cases initially reported to be carcinomas of mixed histologic type. Only two of the latter cases in this study were confirmed upon review, both of which showed endometrioid and clear cell components. In the present analysis these were categorized according to their dominant component (1 case each of CCC and EC). In contrast, 14 carcinomas initially 
reported to be of mixed histologic type were considered to be 'pure' carcinomas, most often HGSC, with variable morphology following review (Table 3, Figs. 4 and 5).

\section{DISCUSSION}

Population-based epidemiological studies can identify factors that may increase or decrease the risks of developing specific types of tumor but analysis is complicated in ovarian neoplasia which encompasses a range of tumor subtypes most of which have distinct clinicopathologic features $(7,8)$. Furthermore, there is evidence that risk factor associations vary according to the histologic subtype of ovarian neoplasia (13). For example, cigarette smoking has been linked to an increased risk of borderline and malignant ovarian mucinous tumors whereas salpingectomy or fallopian tube sterilisation procedures appear protective towards the development of ovarian EC and CCC, both of which are associated with endometriosis (21-23). Bilateral salpingectomy or salpingo-oophorectomy is also protective against HGSC with most such tumors now considered to arise within the fallopian tube (10). It is therefore important that any assessment of risk factors in ovarian neoplasia is subtype-specific, and this in turn necessitates accurate histologic diagnosis. In the present study we have evaluated pathological review as part of this process in a large series of tumors initially categorized as borderline or malignant ovarian neoplasms identified through the WACR.

It is acknowledged that the present pathology review has several limitations. Firstly, a proportion of cases had to be excluded since the initial diagnostic reports could not be accessed, slides were unavailable for review, or because the final diagnosis remained uncertain. We also accept that histologic review was performed in very few borderline tumors and it is likely that at least some cases would now be classified as benign neoplasms with focal proliferative changes, 
based upon the limited extent and mild nature of the atypia (24). Most significantly, in the great majority of cases (approximately 90\%), acceptance of the final diagnosis was based only upon review of the pathology reports, albeit supported by clinical follow-up data and subsequent biopsy findings in many cases. Arguably, the 'gold standard' for pathological assessment would comprise complete histologic review of all tumors, ideally by a panel of pathologists. However, this would be impractical given the number of cases in studies such as this, and it would also be impossible to review those tumors (mainly from the earlier part of the study period) in which the original histologic material was no longer available.

Despite these restrictions, we believe this has been a valuable review process and might serve as a practical approach for similar large epidemiological studies of neoplasia where complete pathological reassessment of all cases is not feasible. The selective histologic review in this study, with re-examination of original slides being prompted by an assessed likelihood of diagnostic error, was supported by the high proportion of amended diagnoses $(82 \%$ of the reviewed excluded cases and $69 \%$ of the reviewed reclassified cases). For example, 33 metastatic tumours were identified most of which had been initially interpreted to be primary ovarian mucinous or endometrioid adenocarcinomas. The histologic difficulty posed by ovarian metastases has been well-documented, and the judicious use of IHC in this setting has also been emphasized $(14,15)$ In the current series IHC was helpful in confirming the metastatic origin of 7 colorectal tumours (including one low-grade appendiceal mucinous neoplasm) and the cervical origin of 3 tumors. Two of the latter occurred in patients with previously treated cervical adenocarcinomas but nonetheless the ovarian tumors were initially interpreted to be independent primary ovarian ECs. These cases presented prior to recent studies which have highlighted the histologic features of cervical adenocarcinoma metastatic to the ovary (25-27), and the correct 
diagnosis was facilitated by additional immunohistochemical analysis including the demonstration of diffuse p16 protein expression in all tumors.

The present review also demonstrated further areas in which there was a significant reclassification of specific tumor subtypes. Notably, only two of 16 carcinomas of mixed histologic type, both of which demonstrated admixed CCC and EC, were confirmed upon review. The remainder, most of which were reported initially as mixed HGSC/EC or mixed HGSC/CCC, were reclassified as pure HGSC with glandular (pseudo-endometrioid) morphology or clear cell change, respectively. As in the case of metastatic neoplasms, this reassessment was often supported by IHC analysis, using the characteristic differential staining patterns of such tumors with WT1, p53, p16, HNF1, napsin A, ARID1A and oestrogen receptor (28). Our findings support recent suggestions that genuine mixed epithelial tumors of the ovary are rare $(9,10,29,30)$.

We also found that many tumors that were initially reported to be undifferentiated carcinomas, or high-grade carcinomas of uncertain origin (primary versus metastatic), were reclassified as HGSC. Likewise, although few tumors in this series had an initial diagnosis of grade $3 \mathrm{EC}$ or TCC, most were reclassified as HGSC upon review with the initial diagnosis being confirmed in only $2 / 6$ and $1 / 4$ cases, respectively. As noted above, the distinction of endometrioid and serous carcinoma can be problematic when the latter exhibits a predominant glandular rather than papillary architecture, but immunophenotypic and molecular data demonstrate the serous nature of the majority of these cases $(9,10,29,30)$. Our findings support the recent study by Lim and colleagues suggesting that ovarian grade $3 \mathrm{EC}$ is uncommon, and that many cases previously categorised as such are in fact HGSC (31). Regarding the consequences of tumor reclassification, it is also noteworthy that the solid (undifferentiated), 
endometrioid-like and transitional-like ('SET') morphologic patterns in HGSC are associated with BRCA1 mutations, whether of germline or somatic origin, with potential implications regarding hereditary predisposition and therapeutic response $(32,33)$.

In summary, we describe the results of pathology report assessment and selective histopathologic review in a population-based series of more than 1500 ovarian tumors identified through a Cancer Registry. The review illustrated specific areas of diagnostic uncertainty, many of which led to case exclusion or to amended diagnosis, thus helping to maintain the accuracy and the utility of the Registry data. We believe this type of review is practical, and the methods could be applied in similar large-scale epidemiological studies to ensure that risk factor assessment in neoplasia is based upon accurate tumor subtyping.

\section{ACKNOWLEDEMENTS}

The authors wish to thank the staff at the WA Data Linkage Branch and the WACR. This research was funded by the Ovarian Cancer Research Foundation (OCRF), Australia, and SJ is funded by a fellowship from the National Health and Medical Research Council of Australia.

\section{REFERENCES}

1. Nakonechny QB, Gilks CB. Ovarian cancer in hereditary cancer susceptibility syndromes. Surg Pathol Clin 2016;9:189-99.

2. Rice MS, Murphy MA, Tworoger SS. Tubal ligation, hysterectomy and ovarian cancer: A meta-analysis. J Ovarian Res 2012;5:13.

3. Stewart LM, Holman CDAJ, Aboagye-Sarfo P, et al. In vitro fertilization, endometriosis, nulliparity and ovarian cancer risk. Gynecol Oncol 2013;128:260-4. 
4. Stewart LM, Holman CDAJ, et al. In vitro fertilization is associated with an increased risk of borderline ovarian tumours. Gynecol Oncol 2013;129:372-6.

5. Tomao F, Russo GL, Spinelle GP, et al. Fertility drugs, reproductive strategies and ovarian cancer risk. J Ovarian Res 2014;7:51.

6. Schuler S, Ponnath M, Engel J, et al. Ovarian epithelial tumors and reproductive factors: a systematic review. Arch Gynecol Obstet 2013;287:1187-204.

7. Cho KR, Shih Ie-M (2009) Ovarian cancer. Annu Rev Pathol 4: 287-313.

8. D'Angelo E, Prat J. Classification of ovarian carcinomas based on pathology and molecular genetics. Clin Transl Oncol 2010;12:783-7.

9. McCluggage WG. My thoughts on and approach to the typing of ovarian carcinomas. J Clin Pathol 2008;61:152-63.

10. Kurman RJ, Shih Ie-M. The dualistic model of ovarian carcinogenesis revisited, revised, and expanded. Am J Pathol 2016;186:733-47.

11. Gurung A, Hung T, Morin J, et al. Molecular abnormalities in ovarian carcinoma: clinical, morphological and therapeutic correlates. Histopathology 2013;62:59-70.

12. Goodman MT, Shvetsov YB. Incidence of ovarian, peritoneal, and fallopian tube carcinomas in the United States, 1995-2004. Cancer Epidemiol Biomarkers Prev 2009;18:132-9.

13. Yang HP, Trabert B, Murphy MA, et al. Ovarian cancer risk factors by histologic subtypes in the NIH-AARP Diet and Health Study. Int J Cancer 2012;131: 938-48.

14. McCluggage WG, Wilkinson N. Metastatic neoplasms involving the ovary: a review with an emphasis on morphological and immunohistochemical features. Histopathology 2005;47:231-47. 
15. Young RH. From Krukenberg to today: the ever present problems posed by metastatic tumors in the ovary. Part 1. Historical perspective, general principles, mucinous tumors including the Krukenberg tumor. Adv Anat Pathol 2006;13:205-27.

16. Shimizu Y, Kamoi S, Amada S, et al. Toward the development of a universal grading system for ovarian epithelial carcinoma: testing of a proposed system in a series of 461 patients with uniform treatment and follow-up. Cancer 1998;82:893-901.

17. Vang R, Shih Ie M, Kurman RJ. Ovarian low-grade and high-grade serous carcinoma: pathogenesis, clinicopathologic and molecular biologic features, and diagnostic problems. $A d v$ Anat Pathol 2009;16:267-82.

18. Stewart CJR, Doherty D, Guppy R, et al. Beta-catenin and E-cadherin expression in stage 1 adult-type granulosa cell tumour of the ovary: correlation with tumour morphology and clinical outcome. Histopathology 2013;62:257-66.

19. Stewart CJR, Alexiadis M, Crook M, et al. An immunohistochemical and molecular analysis of problematic and unclassified ovarian sex cord-stromal tumors. Hum Pathol 2013;44:2774-81. 20. Oost EE, Charles A, Choong CS, et al. Ovarian sex cord-stromal tumors in patients with probable or confirmed germline DICER1 mutations. Int J Gynecol Pathol 2015;34:266-74. 21. Faber MT, Kjaer SK, Dehlendorff C, et al. Cigarette smoking and risk of ovarian cancer: a pooled analysis of 21 case-control studies. Cancer Causes Control 2013: doi:10.1007/s10552013-0174-4.

22. Collaborative Group on Epidemiological Studies of Ovarian Cancer. Ovarian cancer and smoking: individual participant meta-analysis including 28,114 women with ovarian cancer from 51 epidemiological studies. Lancet Oncol 2012;13:936-45. 
23. Mandai M, Yamaguchi K, Matsumura N, et al. Ovarian cancer in endometriosis: molecular biology, pathology, and clinical management. Int J Clin Oncol 2009; 14: 383-91.

24. Seidman JD, Soslow RA, Vang R, et al. Borderline ovarian tumors: diverse contemporary viewpoints on terminology and diagnostic criteria with illustrative images. Hum Pathol 2004 35:918-33.

25. Elishaev E, Gilks CB, Miller D, et al. Synchronous and metachronous endocervical and ovarian neoplasms: evidence supporting interpretation of the ovarian neoplasms as metastatic endocervical adenocarcinomas simulating primary ovarian surface epithelial neoplasms. Am J Surg Pathol 2005;29:281-94.

26. Ronnett BM, Yemelyanova AV, Vang R, et al. Endocervical adenocarcinomas with ovarian metastases. Analysis of 29 cases with emphasis on minimally invasive cervical tumors and the ability of the metastases to simulate primary ovarian neoplasms. Am J Surg Pathol 2008;32:1835-53.

27. Khor TS, Brennan BA, Leung YC, et al. Cervical adenocarcinoma metastatic to the ovary mimicking primary ovarian carcinoma. Pathology 2009;41:397-400.

28. Kaspar HG, Crum CP. The utility of immunohistochemistry in the differential diagnosis of gynecologic disorders. Arch Pathol Lab Med 2015;139:39-54.

29. Kobel M, Kalloger SE, Baker PM, et al. Diagnosis of ovarian carcinoma cell type is highly reproducible. A transCanadian study. Am J Surg Pathol 2010;34:984-93.

30. Mackenzie R, Talhouk A, Eshragh S, et al. Morphologic and molecular characteristics of mixed epithelial ovarian cancers. Am J Surg Pathol 2015;39:1548-57.

31. Lim D, Murali R, Murray M, et al. Morphological and immunohistochemical reevaluation of tumors initially diagnosed as ovarian endometrioid carcinoma with emphasis on high-grade 
tumors. Am J Surg Pathol 2016;40:302-12.

32. Soslow RA, Han G, Park KJ, et al. Morphologic patterns associated with BRCA1 and BRCA2 genotype in ovarian carcinoma. Mod Pathol 2012;25:625-36.

33. Fujiwara M, McGuire VA, Felberg A, et al. Prediction of BRCA1 germline mutation status in women with ovarian cancer using morphology-based criteria. Identification of a BRCA1 ovarian cancer phenotype. Am J Surg Pathol 2012;36:1170-7.

\section{FIGURE LEGENDS}

Figure 1. Outline of review process and case exclusion.

Figure 2. Colonic adenocarcinoma metastatic to the ovary initially interpreted to be primary endometrioid carcinoma. A. The tumor shows well-formed glands and does not involve the ovarian capsule surface (left). B. Higher magnification demonstrating focal cribriform glandular pattern and more prominent mitotic activity and nuclear hyperchromasia than is typical of a lowgrade endometrioid carcinoma. C. Additional immunohistochemistry was characteristic of a primary colorectal neoplasm including diffuse cytokeratin 20 expression (depicted).

Figure 3. Endocervical adenocarcinoma metastatic to the ovary initially interpreted to be primary endometrioid carcinoma. A. The tumor has a partly intracystic papillary architecture. Cribriform glands are focally present (upper left). B. Higher magnification showing glands lined by epithelium exhibiting more prominent nuclear atypia, mitotic and apoptotic activity than is typical of low-grade endometrioid carcinoma. Note focal apical cytoplasmic mucin (left). C. The tumor shows diffuse p16 protein immunoreactivity.

Figure 4. High-grade serous carcinoma initially interpreted to be a mixed endometrioid and serous carcinoma. A. The tumor exhibits glandular (left) and solid (right) growth patterns. There 
are prominent tumour-infiltrating lymphocytes. Immunohistochemistry shows diffuse expression of WT1 (B) and p53 protein (C) in all areas.

Figure 5. High-grade serous carcinoma initially interpreted to be a mixed clear cell and serous carcinoma. A. The tumor shows micropapillary (left and right) and clear cell-like (central) components. B. Higher magnification showing continuity between serous carcinoma (left) and cells with clear cytoplasm (right). Immunohistochemistry throughout was characteristic of highgrade serous carcinoma including WT1 expression in the clear cell areas (depicted). 